IPM/P-2012/027

$\mathrm{IPPP} / 12 / 64$

$\mathrm{DCPT} / 12 / 128$

\title{
Recipes and Ingredients for Neutrino Mass at Loop Level
}

\author{
Yasaman Farzan*(a), Silvia Pascoli ${ }^{\dagger(b)}$, and Michael A. Schmidt ${ }^{\ddagger(c)}$ \\ (a) School of Physics, Institute for research in fundamental sciences (IPM), P.O. Box 19395-5531, Tehran, \\ Iran \\ (b) Institute for Particle Physics Phenomenology (IPPP), University of Durham, Durham DH1 3LE, UK \\ (c) ARC Centre of Excellence for Particle Physics at the Terascale, School of Physics, The University of \\ Melbourne, Victoria 3010, Australia
}

\begin{abstract}
The large hierarchy between the neutrino mass scale and that of the other fermions seems to be unnatural from a theoretical point of view. Various strategies have been devised in order to generate naturally small values of neutrino masses. One of these techniques is neutrino mass generation at the loop level which requires a mechanism, e.g., a symmetry, to forbid the lower order contributions. Here, we study in detail the conditions on this type of symmetries. We put special emphasis on the discrete $Z_{n}$ symmetries as a simple example but our results can be also extended to more general groups. We find that regardless of the details of the symmetry, in certain cases the existence of a lower order contribution to neutrino masses can be determined by the topology of the diagrams with a given number of loops. We discuss the lepton flavor violating rare decays as well as $(g-2)_{\mu}$ in this class of models, which generically appear at the one loop level. Typically the imposed symmetry has important implications for dark matter, with the possibility of stabilizing one or even multiple dark matter candidates.
\end{abstract}

\footnotetext{
*yasaman@theory.ipm.ac.ir

${ }^{\dagger}$ silvia.pascoli@durham.ac.uk

${ }^{\ddagger}$ michael.schmidt@unimelb.edu.au
} 


\section{Introduction}

Explaining nonzero but tiny neutrino masses is one of the most compelling open questions in modern physics. Various beyond Standard Model (SM) theories have been developed to address this question. The most famous mechanism for explaining neutrino masses is the standard (type I) seesaw mechanism [1]. In the standard and simplest realization of this mechanism, the smallness of neutrino masses is connected to the very large mass scale of new SM singlets (right-handed (RH) neutrinos). These new particles, being too heavy, cannot be produced at the LHC or any other man-made or natural environment, (maybe) except for the early universe, making a direct test of these models impossible. With the start of the LHC data release, it is more exciting to move towards models whose new particles are within the reach at the LHC. The smallness of the neutrino masses is not related anymore uniquely to the very heavy mass scale of the RH neutrinos, but requires additional suppressions, e.g., small Yukawa couplings, quasi-conserved lepton symmetries. A very interesting possibility is to forbid neutrino masses at tree level and have them generated at loop-level. The first proposals of this type of radiative neutrino mass models are the Zee model at one loop [2] and the Zee-Babu model at two loop [3].

All seesaw models lead to the effective dimension 5 operator, $(H L)(H L)$. However in general, neutrino masses are not necessarily explained by this dimension 5 operator. Various other $\Delta L=2$ operators can also give rise to neutrino mass. These operators have been classified in [4]. A particular class of these operators are $\left(H^{\dagger} H\right)^{m}(H L)(H L)$ with more than one pair of Higgs fields attached to the corresponding diagram [5]. Recently, there has been a complete classification of one loop diagrams leading to the effective dimension 5 operator in [6] following earlier work [7]. Obviously, the loop generated neutrino masses receive further corrections from renormalization group running, which have been studied for Ma's scotogenic model in [8].

Let us suppose that, thanks to a specific structure of the model, up to the $\mathrm{n}^{\text {th }}$ loop-level, there is no contribution to the neutrino mass matrix. Of course, increasing the loop order will further suppress the neutrino mass. At the three-loop order, with $M_{N E W} \sim 100 \mathrm{GeV}$ and couplings of order of 0.1 , by dimensional analysis the neutrino mass will be in the range $\mathcal{O}\left(\left(g^{2} / 16 \pi^{2}\right)^{3} M_{N E W}\right)-$ $\mathcal{O}\left(\left(g^{2} \log \left(\Lambda / M_{N E W}\right)^{2} / 16 \pi^{2}\right)^{3} M_{N E W}\right) \sim(0.01-1) \mathrm{eV}$ with a cutoff scale $\Lambda \sim(1-10) \mathrm{TeV}$. Such a high value of coupling and low mass scale is very interesting from a phenomenological point of view as it can lead to observable effects in colliders and indirect searches of new physics. Furthermore, the couplings leading to the neutrino mass can also in principle induce Lepton Flavor Violating (LFV) rare decays and a contribution to the anomalous magnetic moment of the muon. The construction of these radiative seesaw models often requires the introduction of an additional symmetry, $G_{\nu}$, forbidding the tree-level contribution as well as contributions from lower loop orders. An interesting consequence of these symmetries is that they stabilize some of the new degrees of freedom and these models can provide a suitable dark matter (DM) candidate [9-14]. Besides a $Z_{2}$ parity, there are several studies involving larger symmetry groups [15-18].

In this paper, we will restrict ourselves to models which lead to the effective dimension 5 
operator. Hence, the left-handed lepton doublets $L$ are the only fermions coupling to the new particles, i.e. leptons act as a portal to the hidden sector. Employing a $G_{\nu}$ symmetry, we restrict the couplings to the form $\mathcal{L}_{Y}=L S_{i} F_{j}$, where $S_{i}\left(F_{j}\right)$ are new scalars (fermions) and forbid couplings of the form $L H F_{j}$ as well as $L L S_{i}$. In this context, we will be general and will not restrict ourselves to the content of a specific model. Our aim is to outline general restrictions and no-go-theorems as a guide to build radiative neutrino mass models. We will consider radiative neutrino mass models up to three loop order. Beyond three loop order, the induced neutrino mass is becoming too small to explain the atmospheric neutrino mass scale. We demand that all the SM particles are invariant under the new symmetry, $G_{\nu}$, but some or all of the new particles transform under $G_{\nu}$. In particular, we assume all new particles that couple directly to $L$ as well as all the new neutral fermions carry a $G_{\nu}$ charge. Moreover, we assume that none of the new scalars receives a vacuum expectation value: that is, $G_{\nu}$ remains unbroken. As a result, the $G_{\nu}$ symmetry forbids a Dirac mass term for the SM neutrinos both at the tree level and at all orders of perturbation theory. The neutrino mass term should be therefore of Majorana type which in turn requires lepton number violation.

For concreteness, we first consider Abelian $Z_{n}$ symmetries (i.e., we take $G_{\nu}=Z_{n}$ ). We classify the emerging topologies up to three loop order and discuss the conditions on the $Z_{n}$ symmetry which forbid all lower loop orders. We then show that some of the results we find also hold valid for a $U(1)$ symmetry and more general symmetries $G_{\nu}$ in sec. 4 .

The paper is organized as follows. In sec. 2, we outline the general setting of the models discussed in the present paper and some general implications for neutrino masses. In sec. 3, we discuss the loop contributions to the neutrino mass matrix and show how the $Z_{n}$ symmetry can forbid lower order contribution to the neutrino mass. In sec. 4 , we discuss how the $Z_{n}$ symmetry can be generalized to other groups. In sec. 5, we discuss the restrictions from LFV rare decays and anomalous magnetic moment of the muon. In sec. 6 , we briefly discuss the implications of the $G_{\nu}$ symmetry for dark matter. In sec. 7 , we summarize our conclusions and briefly comment on implications for LHC signatures.

\section{General setting of the models}

We extend the Standard Model by introducing $N_{S}$ scalars, $S_{i}, i=1, \ldots, N_{S}$, and $N_{F}$ left-handed Weyl fermions, $F_{j}, j=1, \ldots, N_{F}$. We assume that the leptons constitute a portal to the hidden sector via a Yukawa coupling of form

$$
\mathcal{L}_{Y}=\sum_{i}^{N_{S}} \sum_{j}^{N_{F}} g_{i j \alpha} S_{i} F_{j} L_{\alpha}, \quad \text { where } \quad L_{\alpha}=\left(\begin{array}{c}
\nu_{\alpha} \\
l_{\alpha}^{-}
\end{array}\right) .
$$

Throughout the paper, we will adopt a two component notation and write all fields as left-handed Weyl spinors, i.e. in the $\left(\frac{1}{2}, 0\right)$ representation. The product of two Weyl spinors $\xi, \eta$ is therefore defined by $\xi \eta \equiv \xi^{T} c \eta$, where $c$ denotes the charge conjugation matrix (i.e., $c_{11}=c_{22}=0 ; c_{12}=$ 
$\left.-c_{21}=1\right)$. We will use an index-free notation, unless a special discussion of the Lorentz structure is required. Notice that only the combinations of form $F_{j} L_{\alpha}$ in Eq. (1) are allowed. A combination of form $F_{j}^{\dagger} L_{\alpha}$ is forbidden by the Lorentz symmetry. We focus on the neutrino mass generation via coupling Eq. (1) and do not consider other radiative neutrino mass generation mechanisms, e.g., via a coupling of the new particles to $\mathrm{RH}$ charged leptons (See e.g. [9]) or via two $W$ boson exchange [19].

Of course, $\mathcal{L}_{Y}$ should be a $S U(2) \times U(1)$ invariant combination. As a result, the sum of hypercharges of $S_{i}$ and $F_{j}$ is opposite to the hypercharge of $L_{\alpha}$, i.e., $Y_{S_{i}}+Y_{F_{j}}=-Y_{L_{\alpha}}=1$. In case that the hypercharge of the chiral fermions that we are adding is non-zero, anomaly cancellation might require addition of extra chiral fermions. There are various ways to make the combination invariant under $S U(2)$. For example, if $F_{j}$ is a triplet and $S_{i}$ is a doublet, the combination $\epsilon_{a b} S_{i}^{a}\left(F_{j}\right)^{b c} L_{\alpha}^{c}$ is a $S U(2)$ invariant ( $a, b$ and $c$ are $S U(2)$ indices). Our discussion of the implications of the $Z_{n}$ symmetry for loop contributions to the neutrino mass matrix is independent of the behavior of the fields under $S U(2)$ so we shall not specify the behavior of the fields under the electroweak symmetry.

Taking $G_{\nu}=Z_{n}$, the fields in Eq. (1) transform as follows

$$
\begin{aligned}
S_{i} & \rightarrow e^{i \frac{2 \pi}{n} \alpha_{S_{i}} S_{i},} \\
F_{j} & \rightarrow e^{i \frac{2 \pi}{n} \alpha_{F_{j}}} F_{j}, \\
L_{\alpha} & \rightarrow L_{\alpha},
\end{aligned}
$$

such that

$$
\alpha_{S_{i}}+\alpha_{F_{j}} \equiv 0 \quad \bmod n \quad \Leftrightarrow \quad \frac{\alpha_{S_{i}}+\alpha_{F_{j}}}{n} \in \mathbb{Z} \quad \Leftrightarrow \quad \alpha_{S_{i}}+\alpha_{F_{j}} \in n \mathbb{Z} .
$$

If we promote $Z_{n}$ to $U(1)$, the condition in Eq. (3) should be replaced by $\alpha_{S_{i}}+\alpha_{F_{j}}=0$.

Obviously, a Majorana mass term for the fermion $F$ is forbidden, unless the $Z_{n}$ charge of $F$ fulfils $2 \alpha_{F} \equiv 0 \bmod n$. A Weyl fermion $F$ with $2 \alpha_{F} \neq 0 \bmod n$ therefore needs another Weyl fermion, $F^{\prime}$ with $\alpha_{F}=-\alpha_{F^{\prime}}$ to form a Dirac mass term. In case that $F$ and the conjugate of $F^{\prime}$ are in the same representation of $S U(2) \times U(1)$, the mass term will be simply of form $F F^{\prime}$. Anomaly cancellation in this framework will be automatic. If $F$ and $F^{\prime}$ are in different representations of $S U(2) \times U(1)$, anomaly cancellation might require additional chiral fields and a mass term can emerge only after electroweak symmetry breaking. For example, if $F$ is a doublet and $F^{\prime}$ is a singlet, the mass term can originate from a term of form $F F^{\prime} H$.

\section{Loop contributions to neutrino masses}

As discussed earlier, we focus on models within which a Dirac neutrino mass is forbidden by a $Z_{n}$ symmetry and Majorana neutrino masses are produced only at loop level. In subsection 3.1, we make general remarks on the loop contributions to the neutrino mass. In subsection 3.2, we focus specifically on the one-loop contribution. In subsection 3.3, we discuss the conditions for 
constructing a lower loop contribution to the neutrino mass using the propagators and vertices in a general multi-loop diagram, with a specific discussion about the two-loop and three-loop cases in the subsequent subsections. We will analyze the different possible topologies without specifying the SM model charges or even the number of new fields.

\subsection{General remarks on the loop level neutrino mass}

We consider diagrams contributing to neutrino masses and we will indicate the scalar propagators by dashed lines and the fermion propagators by solid ones. In general, the scalar propagator can involve more than one scalar: $\left\langle S_{1} S_{2}^{\dagger}\right\rangle$. Notice that a propagators of form $\left\langle S_{1} S_{2}\right\rangle$ can be rewritten in form of $\left\langle S_{1} S_{3}^{\dagger}\right\rangle$ by redefining $S_{3} \equiv S_{2}^{\dagger}$. Without loss of generality, we will work in a basis with diagonal kinetic terms; as a result, a propagator of form $\left\langle F_{1} F_{2}^{\dagger}\right\rangle$ for $F_{1} \neq F_{2}$ does not exist. The propagators of form $\left\langle F F^{\dagger}\right\rangle$ preserve any $Z_{n}$ symmetry. In general, the fermionic propagator can be either chirality flipping (i.e., of form $\left\langle F_{1} F_{2}^{T} c\right\rangle$ or $\left\langle c F_{1}^{*} F_{2}^{\dagger}\right\rangle$ ) or chirality conserving (i.e., of form $\left.\left\langle F F^{\dagger}\right\rangle\right)$. Propagators of type $\left\langle F_{1} F_{2}^{T} c\right\rangle$ and $\left\langle c F_{1}^{*} F_{2}^{\dagger}\right\rangle$ can result from Dirac or Majorana mass terms.

In order to generate a Majorana mass term $\nu \nu$ for neutrinos, lepton number has to be broken by two units. This can be achieved in various ways. An extensively studied option is to have a Majorana mass term for the new fermions and a lepton number violating mass term of form $m^{2} S^{2} / 2$ for the new scalars. However, the options in general are wider. For example in case of the two-loop diagram in Fig. 5e, if we assign lepton number equal to -1 to $S_{1}$ and $S_{2}$ (or to $F_{1}$ and $F_{2}$ ), lepton number will be broken by two units by the $S_{1} S_{2} S_{3}$ vertex or the $F_{1} F_{2} S_{3}^{\dagger}$ vertex.

A Majorana neutrino mass term $\nu \nu$ can arise only after electroweak symmetry breaking. As we already briefly mentioned, the Weinberg operator is the lowest dimension operator that can induce Majorana mass for neutrinos. Higher dimension operators can also result in neutrino mass. See [4] for a classification of all $\Delta L=2$ operators leading to neutrino masses. However, additional SM fields, which are not charged under $G_{\nu}$ do not affect our discussion of the $G_{\nu}$ symmetry besides generating the neutrino mass at a higher loop order. A particular class of these operators are $\left(H^{\dagger} H\right)^{m}(H L)(H L)$ with more than one pair of Higgs fields attached to the diagram, which have been studied in [5]. In the following, we will concentrate on the simplest origin and only discuss the Weinberg operator $(H L)(H L)$, i.e., a pair of Higgs $H$ being attached to the loop diagram giving mass to neutrinos. Let us discuss each option separately.

- The Higgs can be attached to a vertex of type $S_{1} S_{2} S_{3}$ via a renormalizable coupling $H S_{1} S_{2} S_{3}$, provided that this combination forms a singlet. However, it cannot be attached to a fermionic vertex because the corresponding term in the Lagrangian will be non-renormalizable.

- Let us now discuss the case in which the Higgs field is attached to the propagators. Propagators of form $\left\langle F F^{\dagger}\right\rangle$ and $\left\langle S S^{\dagger}\right\rangle$ cannot break $S U(2) \times U(1)$ but propagators of type $\langle S S\rangle$ 


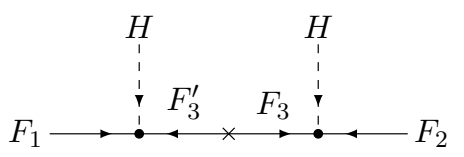

Figure 1: Fermion propagator breaking the hyper-charge by two units

or $\left\langle F F^{T} c\right\rangle$ in principle can do so. Let us take a general propagator of form $\left\langle\phi \psi^{\dagger}\right\rangle$ where $\phi$ and $\psi$ are either both scalars or both left-handed fermions. If $\left\langle\phi \psi^{\dagger}\right\rangle$ breaks hypercharge by one (two) units, electric charge conservation implies $T_{3}(\phi)-T_{3}(\psi)=1(2)$. This means that $\phi$ and $\psi$ cannot be both singlets. A propagator involving only one Dirac field in an $S U(2)$ doublet representation can break hypercharge by at most one unit. However, if we allow more than one field to be involved in the propagators, more possibilities open up. The line shown in Fig. 1 is an example. To have such a line, the required terms are $H F_{3} F_{2}, H F_{3}^{\prime} F_{1}$ and $m_{F_{3}} F_{3} F_{3}^{\prime}$. From now on, for brevity we shall not emphasize on the requirement of mass insertion for a line such as the one denoted by $F_{3}$ and $F_{3}^{\prime}$ in Fig. 1.

Consider a loop that contains $n_{I}$ internal lines plus $n_{S V}$ vertices that involve three scalars. For such a diagram, there are

$$
n_{I}\left[\left(n_{I}+1\right) / 2+n_{S V}\right]+n_{S V}\left(n_{S V}-1\right) / 2
$$

ways to attach the pair of external Higgs fields to the diagram. For example, in case of Fig. $6 \mathrm{~b}$ $n_{I}=8$ and $n_{S V}=3$ which means there are 63 ways of attaching the external pair of Higgs fields. To avoid cluttering the figures with this plethora of possibilities, we do not show the Higgses attached to the diagrams.

\subsection{One loop}

At one loop, there are two possible diagrams, which are not accompanied by a tree-level contribution. They are shown in Fig. 2. Had we included neutral fermions invariant under $G_{\nu}$ or allowed new scalars to develop a VEV, we could have more types of one-loop diagrams [6].

The propagators in the one-loop diagram Fig. 2a are of form $\left\langle S_{1} S_{2}\right\rangle$ and $\left\langle F_{1} F_{2}^{T} c\right\rangle$ where $S_{2}$ and $F_{2}$ may or may not be the same as $S_{1}$ and $F_{1}$, respectively. Let us denote the $Z_{n}$ charge of an arbitrary field $\phi$ by $\alpha_{\phi}$. In order for the propagators to be $Z_{n}$ invariant, the charges of $S_{1}$ and $S_{2}$ as well as the ones of $F_{1}$ and $F_{2}$ have to add up to $0 \bmod n$. The existence of the vertices leads to similar conditions. The following relations need to be satisfied

$$
\alpha_{S_{1}}+\alpha_{S_{2}}, \alpha_{F_{1}}+\alpha_{F_{2}}, \alpha_{S_{1}}+\alpha_{F_{1}}, \alpha_{S_{2}}+\alpha_{F_{2}} \in n \mathbb{Z}
$$

which lead to

$$
\alpha_{S_{1}} \equiv-\alpha_{S_{2}} \equiv \alpha_{F_{2}} \equiv-\alpha_{F_{1}} \quad \bmod n
$$




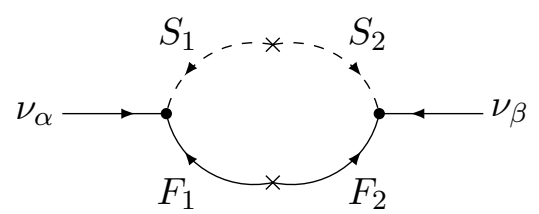

(a) One loop diagram with mass term $S S$ on scalar line

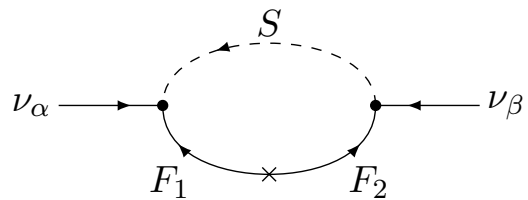

(b) One loop diagram without mass term $S S$ on scalar line.

Figure 2: Effective neutrino mass generation at one loop.

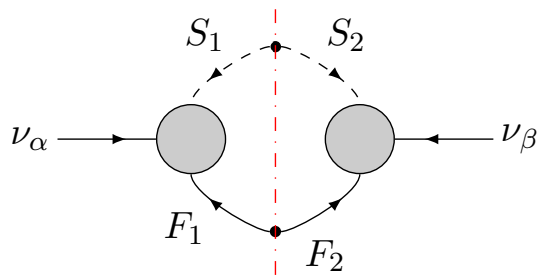

(a)

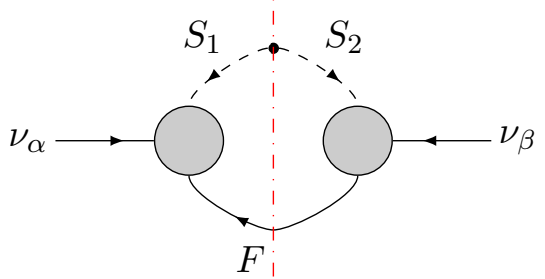

(b)

Figure 3: General examples of loops contributing to the neutrino mass which can be disconnected by cutting a pair of propagators.

In the specific case that the pair of $\left(S_{1}, F_{1}\right)$ is identified with $\left(S_{2}, F_{2}\right)$, we find $2 \alpha_{S_{1}} \equiv 2 \alpha_{F_{1}} \equiv$ $\alpha_{S_{1}}+\alpha_{F_{1}} \equiv 0 \bmod n$ and if there is no other field $\phi$ with non-trivial $Z_{n}$ parity, any choice for $n$ will be equivalent to $n=2$. However, in general when $S_{1} \neq S_{2}$ and $F_{1} \neq F_{2}, n$ might be different from 2.

Let us discuss the special case $S \equiv S_{1}=S_{2}^{\dagger}$, which corresponds to the diagram shown in Fig. 2b. Apparently, in this case the scalar line, $\left\langle S S^{\dagger}\right\rangle$ cannot break $S U(2) \times U(1)$ which means both Higgs fields have to be attached to the fermion line. As discussed earlier, if the fermion line is needed to break hypercharge by two units, it has to involve at least one fermion in addition to $F_{1}$ and $F_{2}$ (see Fig. 1). Notice that in this case no lepton number violating mass insertion of type $m^{2} S^{2} / 2$ is required. Instead, the simultaneous presence of the $S F_{1} L$ and $S^{\dagger} F_{2} L$ vertices and the $F_{1} F_{2}$ mass term breaks lepton number. The Lorentz structure of the Majorana mass term, $\nu \nu$, cannot be created by a fermion propagator of type $\left\langle F F^{\dagger}\right\rangle$ so a mass insertion of the fermionic propagator is required.

The simplest example of this type of models is Ma's scotogenic model [11], which introduces one additional inert Higgs doublet $\eta$ as well as three RH neutrinos $N_{i}$. Neutrino masses are generated with the internal propagators $\left\langle N N^{T} c\right\rangle$ and $\left\langle\eta^{0} \eta^{0}\right\rangle$ with $\eta^{0}$ being the neutral component of the inert Higgs doublet $\eta$. 
The one loop suppression does not suffice to explain the smallness of neutrino masses by itself. A further suppression is needed, which might be due to the smallness of the lepton number violating mass insertions that are indicated by crosses compared to the overall masses of the particles propagating in the loops. Another explanation of the additional suppression might be a sequence of symmetry breaking which naturally suppresses certain couplings (See e.g. [14]).

\subsection{Reduction of multi-loop contribution to one-loop}

Let us consider a general loop contributing to the neutrino mass which can be disconnected by cutting a pair of propagators as shown in Fig. 3a. If, as shown in Fig. 3a, the fermionic propagator is of chirality flipping nature $\left\langle F_{1} F_{2}^{T} c\right\rangle$, the vertices of the following types will be allowed by the $G_{\nu}=Z_{n}$ or $U(1)$ symmetry:

$$
S_{1} L_{\alpha} F_{1} \quad \text { and } \quad S_{2} L_{\beta} F_{2} .
$$

If both the Higgs fields are attached to this pair of lines, these two vertices can be made $S U(2) \times$ $U(1)$ invariant, too. As a result, a one-loop diagram contributing to the neutrino mass with the couplings in Eq. (6) can be formed.

On the other hand, if the propagator is of the chirality-flipping form $\left\langle c F_{1}^{*} F_{2}^{\dagger}\right\rangle$, the vertices $S_{1}^{\dagger} L_{\alpha} F_{1}$ and $S_{2}^{\dagger} L_{\beta} F_{2}$ are allowed by the $G_{\nu}=Z_{n}$ or $U(1)$ symmetry but they may violate $U(1)$ hypercharge. Thus, unlike the previous case, the presence of a one-loop contribution to the neutrino mass is not guaranteed. On the other hand, if the fermionic line is of the chirality-conserving form, i.e., $\left\langle F F^{\dagger}\right\rangle$, see Fig. 3b, vertices in Eq. (6) might violate the $G_{\nu}=Z_{n}$ or $U(1)$ symmetry and again, a one-loop contribution to the neutrino mass does not necessarily exist.

In summary, if there exists a multi-loop contribution to the Weinberg operator, $\left(H L_{\alpha}\right)\left(H L_{\beta}\right)$ compatible with $G_{\nu}=Z_{n}$ or $U(1)$ which can be disconnected by cutting a pair of fermionic and scalar lines, there will be also a one-loop contribution to the neutrino mass provided that (i) both Higgs fields are attached to these two lines; (ii) the fermionic propagator in question is chiralityflipping and of form $\left\langle F_{1} F_{2}^{T} c\right\rangle$.

Let us now consider a general multi-loop diagram of form shown in Fig. 4a in which the internal loop only gives a correction to the wave function of the scalar. Topologically such a diagram is distinguished from the rest by the fact that by cutting the scalar lines directly connected to $\nu_{\alpha}$ and $\nu_{\beta}$, this line will be disconnected. Figs $5 \mathrm{a}, 5 \mathrm{~b}$, and $5 \mathrm{c}$ are examples of such diagrams but Figs. $5 \mathrm{e}$ and $5 \mathrm{f}$ are not. If a contribution of this type exists, the $G_{\nu}=Z_{n}$ or $U(1)$ symmetry allows a term such as $S_{1} S_{2}$, too. Depending on the electroweak behavior of $S_{1}$ and $S_{2}$, this mass term can result from terms such as $\epsilon_{a b} S_{1}^{a} H^{b} S_{2}$ (for singlet $S_{2}$ and doublet $S_{1}$ with $Y_{S_{1}}+Y_{S_{2}}=-1$ ), $\epsilon_{a b} S_{1}^{a} S_{2}^{b}$ (for doublets $S_{1}$ and $S_{2}$ with $Y_{S_{1}}+Y_{S_{2}}=0$ ), $\epsilon_{a b} \epsilon_{c d} S_{1}^{a} H^{b} S_{2}^{c} H^{d}$ (for doublets $S_{1}$ and $S_{2}$ with $Y_{S_{1}}+Y_{S_{2}}=-2$ ) or $S_{1} S_{2}$ (for singlets $S_{1}$ and $S_{2}$ with $Y_{S_{1}}+Y_{S_{2}}=0$ ). As a result, these diagrams are always accompanied by a one-loop diagram.

Let us now discuss diagrams of type in Fig. 4b in which the internal loop gives correction to the 


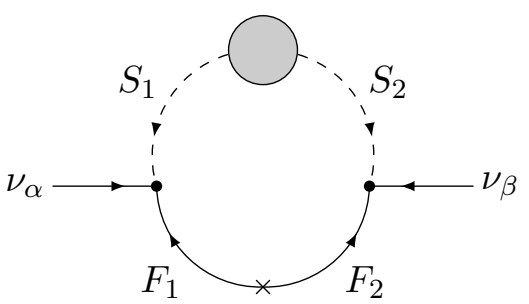

(a) scalar line

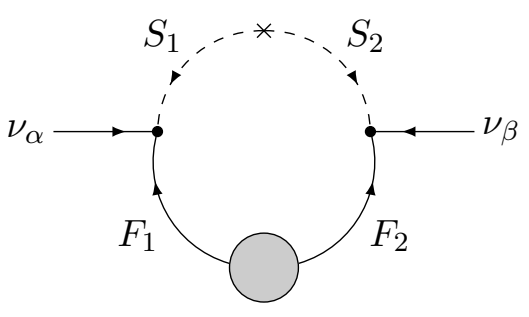

(b) fermion line

Figure 4: Wavefunction renormalization of internal propagators.

wave function of the propagating fermion. Similarly to the correction to the wave function of the scalar propagator, the wave function correction to the fermion propagator can also be written as a $F_{1} F_{2}$ mass term which respects the $G_{\nu}=Z_{n}$ or $U(1)$ symmetry. However, if $F_{1}$ and $F_{2}$ are both electroweak doublets, $F_{1} F_{2}$ will form an electroweak triplet. Thus, two factors of $\langle H\rangle$ are needed to contract it to a $S U(2) \times U(1)$ singlet. In other words, the corresponding term will be nonrenormalizable. Hence, this diagram is not necessarily accompanied by a lower loop contribution depending on the electroweak structure of the fermions.

Let us suppose a coupling of form $g_{\alpha} S F L_{\alpha}$ compatible with $G_{\nu}=Z_{n}$ or $U(1)$ exists. There must be another $F^{\prime}$ with $\alpha_{F^{\prime}}=-\alpha_{F}$ to obtain a Dirac mass term for $F$ (either directly or after electroweak symmetry breaking). The $G_{\nu}=Z_{n}$ or $U(1)$ symmetry does not forbid a term of form $g_{\alpha}^{\prime} S^{\dagger} F^{\prime} L_{\alpha}$. The neutrinos then obtain a Majorana mass proportional to $g_{\alpha} g_{\beta}^{\prime}$ at one loop. In the discussion of higher loops, we implicitly assume that such a possibility is forbidden by other symmetries such as the electroweak symmetry.

\subsection{Two loop}

At two-loop level, there are more possible diagrams. In the following, we again take $G_{\nu}=$ $Z_{n}$ or $U(1)$ and discuss in which cases the symmetries forbid the lower loop contribution to the neutrino mass. In Fig. 5, for the sake of simplicity, some of the scalar lines are marked by a single letter such as $S_{1}$ and $S_{2}$. If the external Higgs is attached to any of these lines, this line will in fact involve more than a single field. When the fermionic propagator involves an even number of fields, the chirality will be flipped because it requires a mass term such as $F_{1} F_{2}$. In the diagrams, the arrow indicates the direction of the flow of the $G_{\nu}$ charge, both for fermion propagator and scalar propagator. Notice that for the fermion lines, the chirality might flip but the direction of the arrow will remain the same. If a chirality flip is required (e.g., Fig. 5f), the fermionic line will involve the fermion, $F$ and its partner $F^{\prime}$ with opposite $G_{\nu}$ charge that together form a Dirac mass. This simplified way of marking does not generally affect our discussion below. We will be more specific when it does. 


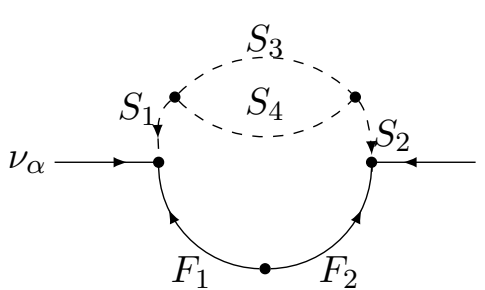

(a)

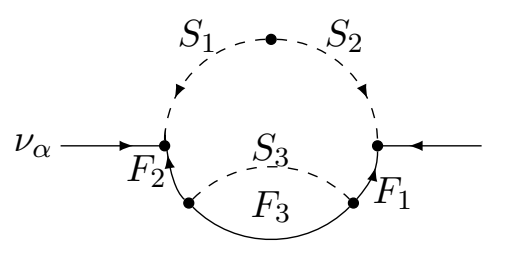

(d)

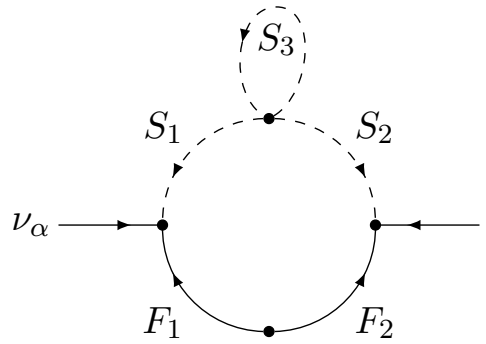

(b)

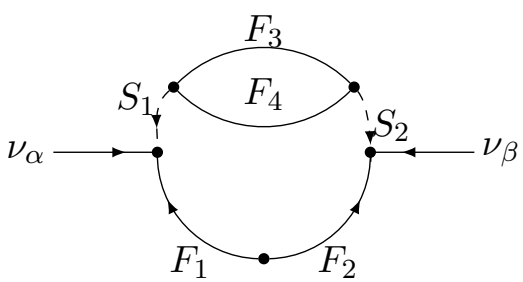

(c)

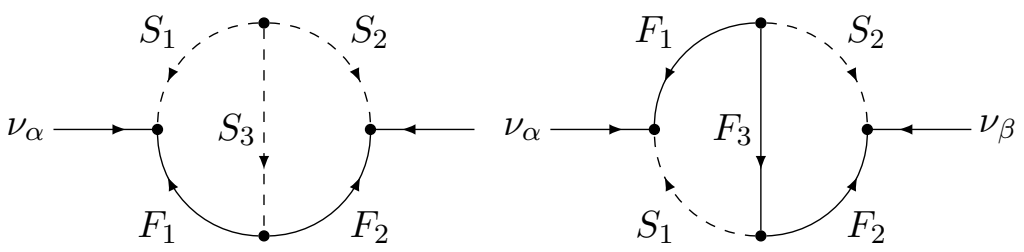

(e)

(f)

Figure 5: Two loop diagrams

As discussed in the previous section, the diagrams 5a, 5b, and 5c are always accompanied by a one-loop diagram but this is not necessarily the case for the diagram in Fig. 5d, as it has been discussed in the previous section. The diagrams $5 \mathrm{e}$ and $5 \mathrm{f}$ cannot be reduced to a one loop diagram, as long as $S_{3}$ and $F_{3}$ transform non-trivially under $G_{\nu}=Z_{n}$ or $U(1)$, so they can give the dominant contribution to neutrino mass.

\subsection{Three loop}

Before starting the discussion of the three-loop diagrams, we emphasize that the comment in the first paragraph of the previous section on marking the propagators applies here, too. The threeloop diagrams contributing to the neutrino mass can be divided into three categories: (i) diagrams in which the inner loops correct the wave function of the internal lines. Such diagrams are already discussed in sect. 3.3. (ii) Planar diagrams shown in Figs. 6, 7, 8 and 9. We shall discuss all these diagrams in detail below in context of $G_{\nu}=Z_{n}$ and $G_{\nu}=U(1)$. (iii) Non-planar diagrams shown in Fig. 10 which will be discussed in detail in this section in context of $G_{\nu}=Z_{n}$ and briefly for $G_{\nu}=U(1)$. Although at first sight it seems there are four non-planar three-loop diagrams contributing to the neutrino mass, only two are independent. As demonstrated in Fig. 10 twisting the vertices denoted by $P_{1}$ and $P_{3}$, the diagrams on the left- and right-hand sides of Fig. 10 convert into each other. There are therefore only two distinct non-planar diagrams.

The $G_{\nu}=Z_{n}$ or $G_{\nu}=U(1)$ symmetries do not forbid lower order loop contributions for any of the planar diagrams besides the one in Fig. 9, but the pattern of electroweak symmetry breaking 


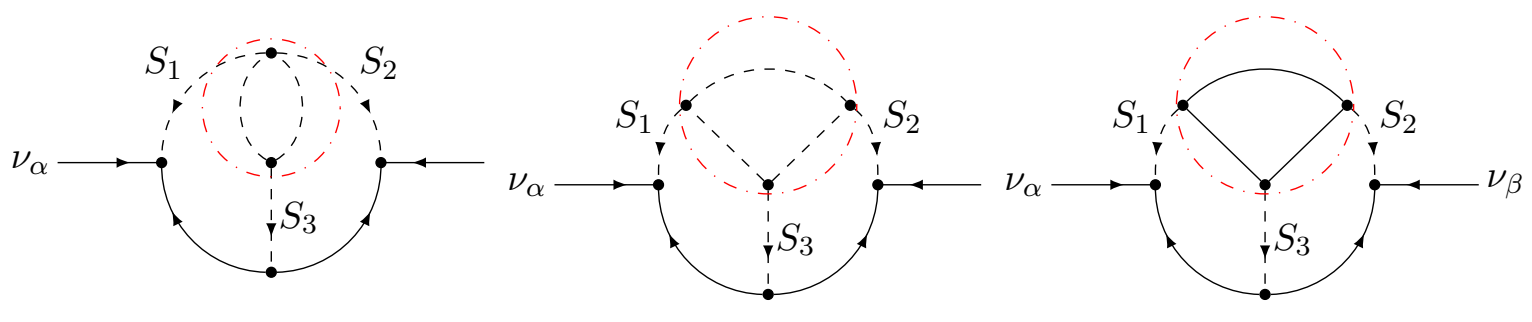

(a)

(b)

(c)

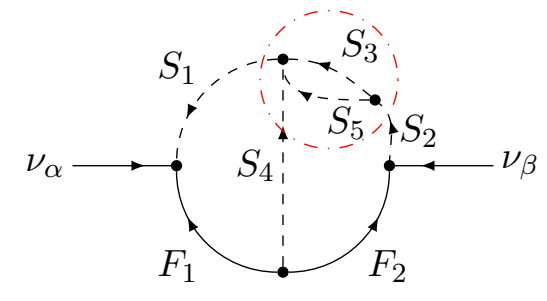

(d)

Figure 6: Planar three loop diagrams.

as well as the requirement of chirality flipping might prevent some. Let us discuss this possibility in detail. In Fig. 6 and Fig. 7, the internal loop in the red dashed dotted circle can be replaced by an effective $G_{\nu}$ conserving vertex. Let us first consider the diagrams in Fig. 6. For a Lagrangian symmetric under $G_{\nu}=Z_{n}$ (or under $G_{\nu}=U(1)$ ), the presence of these diagrams is possible only if $\alpha_{S_{i}}$ associated with $S_{1}, S_{2}$ and $S_{3}$ add up to an integer times $n$ (or add up to zero). Thus, the $G_{\nu}$ invariant Lagrangian contains vertices of type $S_{1} S_{2} S_{3}+$ h.c. unless it is forbidden by some other symmetry. In particular let's consider the $S U(2) \times U(1)$ symmetry: each line might involve more than one field with different $S U(2) \times U(1)$ quantum numbers and we should specify the fields that directly leave the red dashed dotted circle. In case that $Y_{S_{1}}+Y_{S_{2}}+Y_{S_{3}}=0$ or -1 , the corresponding vertex can be just $S_{1} S_{2} S_{3}+$ h.c. or $S_{1} S_{2} S_{3}\langle H\rangle+$ h.c., respectively. However, there is no renormalizable vertex of form $S_{1} S_{2} S_{3}$ if $Y_{S_{1}}+Y_{S_{2}}+Y_{S_{3}}<-1$ and no corresponding two-loop diagram. Similarly, in case of the diagrams in Fig. 7, the loop in the dashed dotted red circle can be also replaced by a renormalizable $Z_{n}$ invariant vertex of form $S_{1}^{\dagger} F_{1}^{\dagger} F_{2}^{\dagger}$ unless $Y_{S_{1}}+Y_{F_{1}}+Y_{F_{2}} \neq 0$. Notice that we implicitly assume that both left-handed fields $F_{1}$ and $F_{2}$ leave the red dashed dotted circle; i.e., the corresponding effective vertex is of form $S_{1}^{\dagger} F_{1}^{\dagger} F_{2}^{\dagger}$ rather than $S_{1}^{\dagger} F_{2}^{\dagger} F_{1}$ or $S_{1}^{\dagger} F_{1}^{\dagger} F_{2}$ which are forbidden by Lorentz structure. Let us consider the loop on the right-hand side in the diagrams of Fig. 8. If, as indicated in the figures, the fermion marked with $F$ enters this loop, the loop can be replaced by a renormalizable $Z_{n}$ invariant Yukawa coupling of form $S^{*} F L_{\beta}$, which conserves hypercharge if $Y_{S}=Y_{F}-1$.

The implication of $Z_{n}$ for Fig. 9 as well as the non-planar diagrams in Fig. 10 is more complicated. In particular, $Z_{n}$ does not always allow them to be accompanied by a dominating two loop 


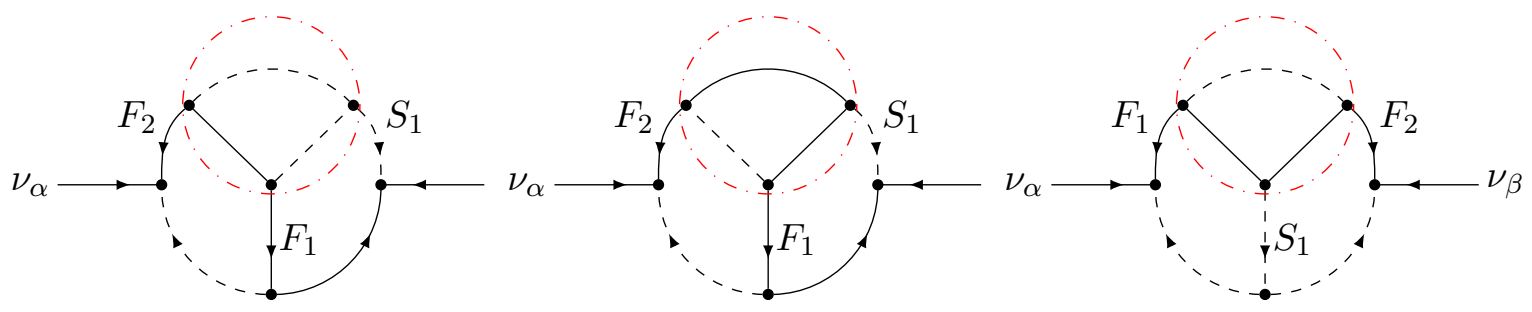

(a)

(b)

(c)

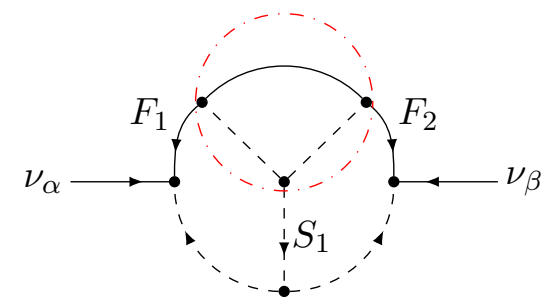

(d)

Figure 7: Planar three loop diagrams.

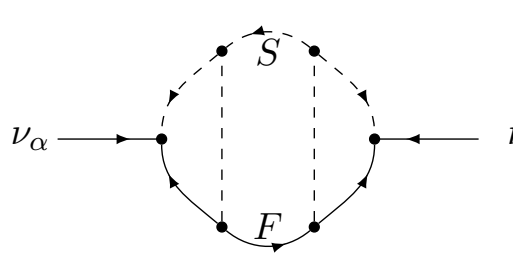

(a)

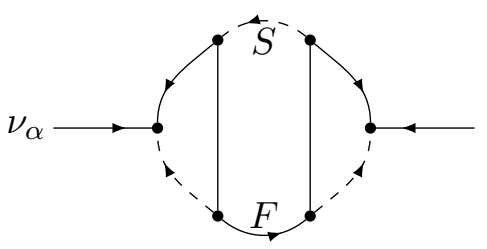

(b)

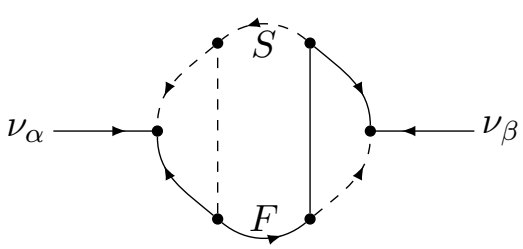

(c)

Figure 8: Planar three loop diagrams.

contribution to the neutrino mass. Let us first consider diagram 9. The $Z_{n}$ symmetry implies

$$
\begin{array}{ll}
\alpha_{F_{1}}=-\alpha_{S_{1}}+n k_{1} & \alpha_{F_{3}}=\alpha_{S_{3}}-\alpha_{S_{1}}+n k_{3} \\
\alpha_{F_{2}}=-\alpha_{S_{2}}+n k_{2} & \alpha_{S_{4}}=\alpha_{S_{1}}+\alpha_{S_{2}}-\alpha_{S_{3}}+n k_{4}
\end{array}
$$

where $k_{i}$ are arbitrary integers. We are interested to find out whether there is a $Z_{n}$ symmetry with certain $\alpha_{\phi}$ assignment which is compatible with the diagram in Fig. 9, i.e., satisfies Eqs. (7) but forbids lower loop contributions. To answer this question, we have solved equations (7) under the condition that none of the one- and two-loop diagrams respectively in Fig. 2 and Fig. 5, is allowed by the $Z_{n}$ symmetry. The values of $\alpha_{S_{i}}$ and $\alpha_{F_{j}}$ can be set such that the $Z_{n}$ symmetry forbids the lower orders of contributions to the neutrino mass for $n \geq 16$. One example is the charge assignment

$$
\alpha_{S_{1}}=1 \quad \alpha_{S_{2}}=3 \quad \alpha_{S_{3}}=9 \quad \alpha_{S_{4}}=11 \quad \alpha_{F_{1}}=15 \quad \alpha_{F_{2}}=13 \quad \alpha_{F_{3}}=8
$$




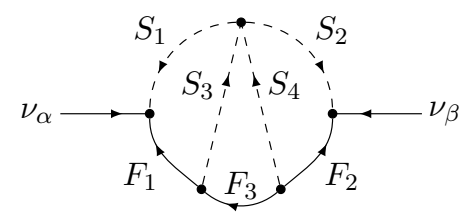

Figure 9: Planar three loop diagrams.
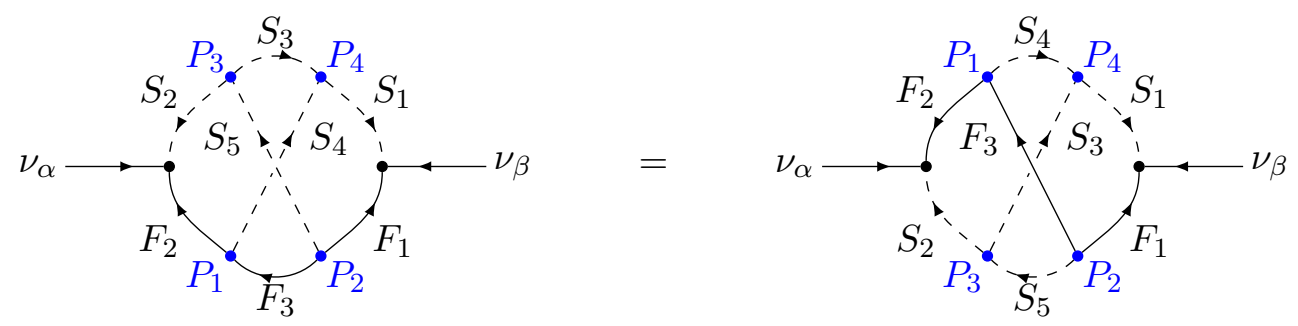

(a)
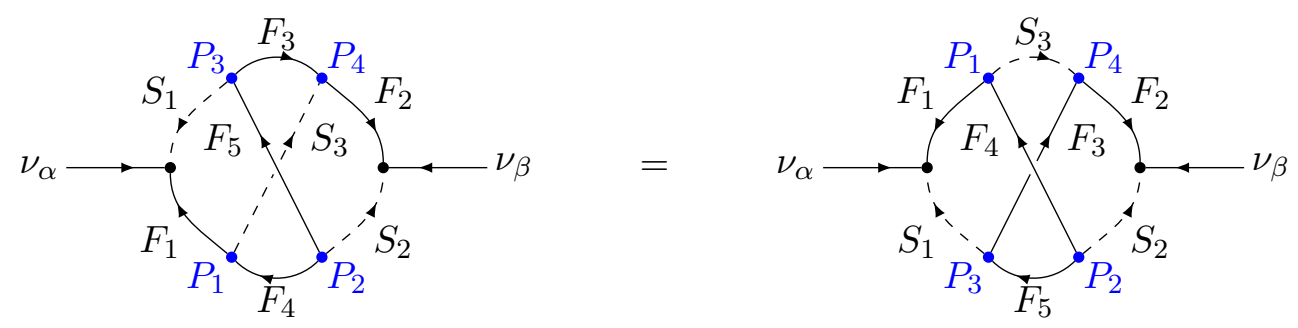

(b)

Figure 10: Non-planar three loop diagrams

for $Z_{16}$. However, for smaller values of $n$ such an assignment is not possible.

Similarly in case of the non-planar diagrams, for certain assignments of $\alpha_{S_{i}}$ and $\alpha_{F_{j}}$, the $Z_{n}$ symmetry forbids all diagrams with a lower loop order. Let us consider diagram Fig. 10a. The $Z_{n}$ symmetry leads to the following relations for the different fields propagating inside the loops:

$$
\begin{array}{ll}
\alpha_{F_{1}}=-\alpha_{S_{1}}+n k_{1} & \alpha_{S_{3}}=\alpha_{S_{5}}-\alpha_{S_{2}}+n k_{4} \\
\alpha_{F_{2}}=-\alpha_{S_{2}}+n k_{2} & \alpha_{S_{4}}=\alpha_{S_{1}}+\alpha_{S_{2}}-\alpha_{S_{5}}+n k_{5} \\
\alpha_{F_{3}}=\alpha_{S_{1}}-\alpha_{S_{5}}+n k_{3} . &
\end{array}
$$

We have found that the smallest value of $n$ for which the $Z_{n}$ symmetry forbids lower order loop contribution to the neutrino mass is $n=16$. One particular example is

$$
\alpha_{S_{1}}=2 \quad \alpha_{S_{2}}=6 \quad \alpha_{S_{3}}=13 \quad \alpha_{S_{4}}=5 \quad \alpha_{S_{5}}=3 \quad \alpha_{F_{1}}=14 \quad \alpha_{F_{2}}=10 \quad \alpha_{F_{3}}=15
$$




\begin{tabular}{c|cccccccc} 
Fig. 10a & $S_{1}$ & $S_{2}$ & $S_{3}$ & $S_{4}$ & $S_{5}$ & $F_{1}$ & $F_{2}$ & $F_{3}$ \\
\hline Fig. 10b & $F_{2}$ & $F_{1}$ & $F_{5}$ & $F_{4}$ & $F_{3}$ & $S_{2}$ & $S_{1}$ & $S_{3}$
\end{tabular}

Table 1: Translation table between Fig. 10a and Fig. 10b.

for $Z_{16}$. That is for $n<16$ any possible assignment of $\alpha_{S_{i}}$ and $\alpha_{F_{i}}$ will also lead to a dominant lower loop diagram. In this analysis, only the $Z_{n}$ symmetry is considered. Of course, depending on the field content of the specific model, the pattern of the hypercharge breaking as well as the form of chirality flipping might also forbid the lower loop contribution.

A similar analysis is performed for the diagram in Fig. 10b. To be compatible with the $Z_{n}$ symmetry, the following set of equations has to be satisfied

$$
\begin{array}{ll}
\alpha_{F_{1}}=-\alpha_{S_{1}}+n k_{1} & \alpha_{F_{4}}=-\alpha_{S_{1}}+\alpha_{S_{3}}+n k_{4} \\
\alpha_{F_{2}}=-\alpha_{S_{2}}+n k_{2} & \alpha_{F_{5}}=\alpha_{S_{1}}-\alpha_{S_{2}}-\alpha_{S_{3}}+n k_{5} \\
\alpha_{F_{3}}=-\alpha_{S_{2}}-\alpha_{S_{3}}+n k_{3} &
\end{array}
$$

for any integers $k_{i}$. Here, $n=16$ is again the smallest value of $n$ for which $Z_{n}$ forbids a lower loop contribution to the neutrino mass. One example of charge assignment for $Z_{16}$ forbidding lower loop contributions is

$$
\alpha_{S_{1}}=2 \quad \alpha_{S_{2}}=6 \quad \alpha_{S_{3}}=3 \quad \alpha_{F_{1}}=14 \quad \alpha_{F_{2}}=10 \quad \alpha_{F_{3}}=7 \quad \alpha_{F_{4}}=1 \quad \alpha_{F_{5}}=9 .
$$

Notice that all three of the three loop diagrams require $n \geqslant 16$ to forbid lower order loop diagrams. We will first show that the conditions for Fig. 10a and Fig. 10b are equivalent. If we identify the charges in Eq. (9), as indicated in Tab. 1, it is straightforward to show, that we obtain a set of equations, which is equivalent to Eq. (11) and vice versa. Note that we are identifying the charges of the fermions in one diagram with the ones of the scalars in the other diagram and vice versa. Remember that to form a Dirac mass term for the new fermions, we have to introduce a partner for each fermion so the charges of scalars and fermions may be treated on an equal footing. As a result, the equivalence of the conditions for the absence of lower loop contributions directly follows. The equivalence of charge assignments also implies that for a given $n \geqslant 16$, there must be exactly the same number of possible charge configurations for each diagram that forbids the lower order loops. In fact, solving the equations for $n=16$, we find that there are exactly 32 possible charge configurations for each of the diagrams Fig. 10a and Fig. 10b that forbid lower order contributions.

It is straightforward to verify that after replacing

$$
\alpha_{S_{3}} \leftrightarrow \alpha_{S_{5}} \quad \alpha_{F_{3}} \leftrightarrow-\alpha_{F_{3}},
$$

Eqs. (7) will be equivalent to the first four equations in Eq. (9). As a result, if for a given $n$ there is an assignment of charges for the field content of Fig. 10a that forbids lower loop contributions to the neutrino mass, the corresponding assignment for Fig. 9 will also forbid lower order contributions. 
However, the opposite statement is not valid. In fact, the presence of an extra scalar field in case of Fig. 10a gives more freedom to construct more possible lower loop diagrams. Setting $n=16$, we find 112 solutions for Fig. 9 that forbid lower loop contributions but only 32 such solutions for diagrams in Fig. 10a.

In this discussion, we have assumed that each fermionic (scalar) propagator has an independent $\alpha_{F_{i}}\left(\alpha_{S_{j}}\right)$ value. The relation between $\alpha_{F_{i}}$ and $\alpha_{S_{j}}$ comes from the requirement that this diagram respects $Z_{n}$. However, within specific models, there might be more restrictions. For example, let us assume that $S_{1}$ and $S_{2}$ in diagram Fig. 10a are the same fields. We then conclude that for any value of $n$ and any choice of $\alpha_{F_{i}}$ and $\alpha_{S_{j}}$ (provided that $\alpha_{S_{1}}=\alpha_{S_{2}}$ ), the $Z_{n}$ symmetry allows the diagram in Fig. 10a to be accompanied with two diagrams of form in Fig. 5e with the following replacements of the fields in Fig. 5e: $\left(S_{1}, S_{2}, S_{3}, F_{1}, F_{2}\right) \rightarrow\left(S_{1}, S_{3}, S_{5}^{*}, F_{1}, F_{3}\right)$ or $\left(S_{1}, S_{2}, S_{3}, F_{1}, F_{2}\right) \rightarrow\left(S_{1}, S_{3}^{*}, S_{4}^{*}, F_{1}, F_{3}^{\prime}\right)$ where $F_{3}^{\prime}$ is the Weyl fermion with charge opposite to that of $F_{3}$ that together form a Dirac mass term $F_{3} F_{3}^{\prime}$. Depending on the field content of the model, it is possible that one or both of these two-loop diagrams are forbidden by $S U(2) \times U(1)$ or by pattern of chirality flipping. For example, if in Fig. 10a, one of the Higgs fields is attached to $S_{4}$ and the other to $S_{5}$, neither of these two-loop diagrams can exist because each violates hypercharge only by one unit.

Let us now briefly discuss the possibility of replacing $Z_{n}$ with a continuous $U(1)$. In this case setting $n=0$, relations in Eqs. (9) and (11) remain valid. For a $U(1)$ with general $\alpha_{S_{i}}$ and $\alpha_{F_{j}}$, the three-loop non-planar diagrams and the planar diagram in Fig. 9 cannot be accompanied with a lower loop contribution unless in very specific cases. For example, in the specific case that $\alpha_{S_{4}}=-\alpha_{S_{5}}$, there might be also a one-loop contribution to the neutrino mass accompanying the diagram in Fig. 10a.

\section{General symmetry}

In previous sections, we focused on the implications of $G_{\nu}=Z_{n}$ or $U(1)$ symmetry on the neutrino mass generation at loop levels. As we shall discuss below, some of these discussions can be applied for a general symmetry group $G_{\nu}$. We will make a further generalization in this section. Motivated by the DM models, in the previous sections, we have assumed that the SM particles are all invariant under the $G_{\nu}$ symmetry. In this section, we discuss the consequences of relaxing this assumption. We will still assume that none of the scalar fields which non-trivially transform under $G_{\nu}$ (i.e., are not invariant under $G_{\nu}$ ) receives a VEV. Thus, the SM Higgs is invariant under $G_{\nu}$.

Let us reconsider coupling (1) and review the results that we found in the previous sections.

- As long as all new neutral fermions non-trivially transform under $G_{\nu}$, the Dirac mass term for neutrinos will be forbidden by this symmetry to all orders in perturbation theory.

- Consider a general $n$-loop diagram contributing to neutrino mass. Suppose that there is a 
sub-diagram within this diagram that absorbs scalar lines $S_{1}, S_{2}$ and $S_{3}$. The existence of such a sub-diagram implies that a combination of form $S_{1} S_{2} S_{3}$ is invariant under $G_{\nu}$. If no Higgs VEV is attached to this sub-diagram, it means this combination is also invariant under electroweak symmetry and this term is allowed in the Lagrangian. Thus, there should be a lower order contribution to the neutrino mass where this sub-diagram is replaced by the $S_{1} S_{2} S_{3}$ vertex. Similarly, if there is only one Higgs VEV attached to this sub-diagram, a renormalizable coupling of form $S_{1} S_{2} S_{3} H$ exists in the Lagrangian which can lead to a lower order contribution to the neutrino mass. However, if both Higgs VEVs of the Weinberg operator are attached to the sub-diagram, the corresponding electroweak vertex will not be renormalizable. Similarly, there might be a sub-diagram in which two fermions $\left(F_{1}\right.$ and $F_{2}$ ) and one scalar $\left(S_{1}\right)$ leave the sub-diagram. Examples are shown in Fig. 7, where the sub-diagram is inside the red circle. Regardless of the details of $G_{\nu}$ and whether the SM particles are invariant under it or not, a vertex of form $F_{1} F_{2} S_{1}$ is invariant under $G_{\nu}$. If it is also invariant under electroweak symmetry (i.e., if no Higgs VEV is attached to the sub-diagram) this renormalizable term will be present in the Lagrangian. This means this diagram is accompanied by a lower order diagram in which the sub-diagram is replaced by vertex of form $F_{1} F_{2} S_{1}$.

- Let us now consider a general diagram that contains a sub-diagram which is a correction to the self-energy of a scalar line, that is the external lines that are attached to this sub-diagram are two scalars $S_{1}$ and $S_{2}$ (See Fig. 4a). The $S_{1} S_{2}$ term will be $G_{\nu}$ invariant and in case that less than three Higgs VEVs are attached to this sub-diagram, it can be made electroweak invariant by adding appropriate number of Higgs fields (i.e., $S_{1} S_{2}, S_{1} S_{2} H$ or $S_{1} S_{2} H H$ ). The diagram is accompanied by another one in which the sub-diagram is replaced by the corresponding renormalizable vertex. Similar consideration holds for a sub-diagram giving correction to the fermion self energy but in this case more than one Higgs fields cannot

be added otherwise the corresponding term will be non-renormalizable. Notice that this consideration holds valid regardless of the details of the $G_{\nu}$ symmetry and the behavior of the SM fermions under this symmetry.

- The theorem of section 3.3, regarding diagrams of form shown in Fig. 3 holds valid for a general $G_{\nu}$ independent of the behavior of the SM fermion under the symmetry transformation.

- As shown for the special case of $G_{\nu}=Z_{n}$, the $G_{\nu}$ symmetry can forbid lower order contribution for the two-loop diagram of topology shown in Fig. 5e and Fig. 5f, as well as the three-loop diagrams of topology in Figs. 9 and 10.

\section{Lepton flavor violation}

After discussing the general structure of the different diagrams leading to neutrino masses, we will discuss lepton flavor violating rare decays which have proven to lead to strong constraints on 


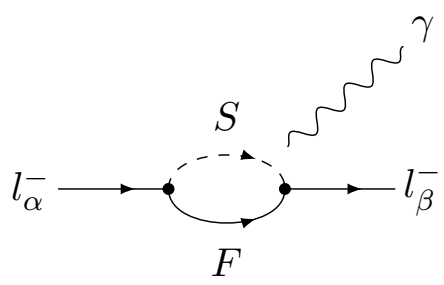

Figure 11: LFV diagram. The photon is emitted either from the initial or final state or from the charged particle in the loop.

radiative neutrino mass models. The general Yukawa coupling in Eq. (1) includes LFV couplings of left-handed charged leptons, $l_{\alpha}^{-}, g_{i j \alpha} s_{i} f_{j} l_{\alpha}^{-}$where $s_{i}$ and $f_{j}$ are components of the multiplets $S_{i}$ and $F_{j}$ such that the following relation holds valid between their electric charges:

$$
Q_{s_{i}}+Q_{f_{j}}=-Q_{l_{\alpha}^{-}}=1 .
$$

This LFV coupling will lead to LFV rare decay $l_{\alpha}^{-} \rightarrow l_{\beta}^{-}+\gamma$ as shown in Fig. 11 . Note that in contrast to the case of contributions to the neutrino masses, the LFV rare decays are generically allowed at one loop order unless there is a flavor symmetry forbidding the one loop contribution; see e.g. [20]. Neglecting the corrections of order of $\left(m_{l_{\beta}} / m_{l_{\alpha}}\right)^{2}$, the decay rate is [21]

$$
\Gamma\left(l_{\alpha}^{-} \rightarrow l_{\beta}^{-}+\gamma\right)=\frac{\alpha_{Q E D} m_{l_{\alpha}^{-}}^{5}}{\left(384 \pi^{2}\right)^{2}}\left|\sum_{i j} \frac{g_{i j \alpha} g_{i j \beta}^{*}}{m_{s_{i}}^{2}}\left(Q_{f_{j}} J\left[\frac{m_{f_{j}}^{2}}{m_{s_{i}}^{2}}\right]+I\left[\frac{m_{f_{j}}^{2}}{m_{s_{i}}^{2}}\right]\right)\right|^{2},
$$

where

$$
I[t]=\frac{2 t^{2}+5 t-1}{(t-1)^{3}}-\frac{6 t^{2} \ln t}{(t-1)^{4}}
$$

and

$$
J[t]=\frac{3 t+3}{(t-1)^{2}}-\frac{6 t \ln t}{(t-1)^{3}} .
$$

$I(t)$ and $J(t)$ are monotonously decreasing functions with the following values:

$$
\begin{array}{lll}
I(0)=1 & I(1)=1 / 2 & I(t) \stackrel{t \rightarrow \infty}{\longrightarrow} \frac{2}{t} \\
J(0)=3 & J(1)=1 & J(t) \stackrel{t \rightarrow \infty}{\longrightarrow} \frac{3}{t} .
\end{array}
$$

Notice that this relation holds regardless of the representation of the electroweak symmetry to which the new particles belong. Larger representations can lead to several different possible loop diagrams.

In the case that the neutrino mass originates from the three-loop contribution, the coupling should be of order one for $m_{N E W} \sim 100 \mathrm{GeV}$ to account for $m_{\nu} \sim \sqrt{\Delta m_{\text {atm }}^{2}}$. This will lead to 
$\operatorname{Br}(\mu \rightarrow e \gamma)$ exceeding the present bound. However, there are ways to avoid these bounds making use of a particular flavor structure. In the following, we explain a simple and natural solution. To reproduce two nonzero neutrino mass eigenvalues, more than one pair of $(S, F)$ coupled to $L$ is required, which we will call $\left(S_{1}, F_{1}\right)$ and $\left(S_{2}, F_{2}\right)$. Let us suppose that $F_{1}$ only couples to $L_{e}$ while $F_{2}$ couples to $L_{\mu}$ and $L_{\tau}$. That is $g_{11 \mu}=g_{11 \tau}=g_{22 e}=0$. In this case, the couplings conserve $L_{e}$ so $\mu \rightarrow e \gamma$ will be absent. As it is well know [22], the conservation of $L_{e}$ leads to a vanishing first row and column of the neutrino mass matrix, i.e., $m_{e \alpha}=0$ for $\alpha=e, \mu, \tau$, and can therefore only serve as a leading order approximation of neutrino masses. If $L_{e}$ is softly broken by trilinear scalar couplings, the vanishing elements can be reproduced and the observed neutrino mass pattern can be reconstructed. Obviously, the breaking introduces $\mu \rightarrow e \gamma$, but it is controlled by the smallness of the symmetry breaking and will not be dangerous.

The new coupling can lead to a new contribution to anomalous magnetic moment of muon as follows

$$
\frac{(g-2)_{\mu}}{2}=\frac{m_{\mu}^{2}}{192 \pi^{2}} \sum_{i j} \frac{\left|g_{i j \mu}\right|^{2}}{m_{s_{i}}^{2}}\left(Q_{f_{j}} J\left[\frac{m_{f_{j}}^{2}}{m_{s_{i}}^{2}}\right]+I\left[\frac{m_{f_{j}}^{2}}{m_{s_{i}}^{2}}\right]\right) .
$$

Notice that for $m_{S}, m_{F} \sim 100 \mathrm{GeV}$ and $g \sim 0.5$, this contribution can explain the observed anomaly.

\section{Dark matter}

The symmetry required to suppress neutrino masses can have important implications for dark matter. Since we have taken the SM particles to be invariant under the $G_{\nu}$ symmetry, this symmetry protects the lightest new particle from decay and the latter constitutes a potential DM candidate. Depending on the exact form of the symmetry, there might be several stable particles and multiple DM components. We briefly discuss the main features of these models. For a detailed discussion of specific realizations, we refer the reader to Ref. [16] in the case of an abelian $Z_{n}$ symmetry and Ref. [17] for an explicit construction of a $D_{3}$ model. We will briefly summarize the main points. In the case of direct product groups, i.e. groups $G$ which can be written as $G_{1} \times G_{2}$ with two arbitrary groups $G_{i}$, there can be two DM candidates, given by the two lightest particles transforming non-trivially under each of the two group factors. This happens for example for a model based on the Abelian finite group $Z_{6} \cong Z_{3} \times Z_{2}$ containing two fields with $Z_{6}$ charges equal to +2 and +3 , respectively. More generally, for every subgroup $H$ of $G$, the lightest particle transforming non-trivially under the subgroup might potentially be stable and a DM candidate. This may lead to a plethora of DM candidates. In the case of finite abelian groups, there is a complete classification in terms of direct products of $Z_{p_{i}^{n_{i}}}$ factors with $p_{i}$ being prime numbers and $n_{i}$ natural numbers. Each factor of order $p^{n}$ has non-trivial subgroups $Z_{p^{m}}$ with $0<m<n$. Hence, there are potentially $\sum_{i} n_{i}$ DM candidates, one for each non-trivial subgroup, depending on the mass spectrum. As an example, let us consider a model with $Z_{4}=Z_{2^{2}}$ symmetry containing two fields which under $Z_{4}$ transform as follows: $\phi_{1} \rightarrow e^{i \pi / 2} \phi_{1}, \phi_{2} \rightarrow e^{i \pi} \phi_{2}$. The $Z_{4}$ symmetry 
contains a $Z_{2}$ subgroup under which $\phi_{1} \rightarrow-\phi_{1}$ and $\phi_{2} \rightarrow \phi_{2}$. For $m_{\phi_{1}}<m_{\phi_{2}} / 2, \phi_{1}$ is the only DM candidate, since $\phi_{2}$ can decay into $\phi_{1} \phi_{1}$ via the coupling $\phi_{1} \phi_{1} \phi_{2}$. In case $m_{\phi_{2}} / 2<m_{\phi_{1}}$, both fields $\phi_{i}$ will be stable and therefore DM candidates.

Generally, in presence of multiple DM candidates coannihilation will take place. However, in the models considered, particles belonging to different factors of a direct product group will not coannihilate. In the following, we will restrict ourselves to the simplest case of an abelian group, which can be written in terms of a direct product of groups without a proper subgroup, i.e. there is no coannihilation between the DM candidates. In order to prevent having a charged DM candidate, the stable particles must be either neutral or in case that they are charged, their annihilation cross section should be much larger than $10^{-36} \mathrm{~cm}^{2}$. We focus on the SM gauge, Yukawa interactions as well as annihilation via the Higgs portal, considering each possibility one by one. In principle, there might be a new gauge interaction contributing to DM annihilation but we will not discuss this additional extension of the models. We will mainly consider the cases in which the DM belongs to a doublet, is a singlet or combination of the two.

\section{Annihilation via $Z$ boson exchange}

First, let us consider the case in which the dark matter is the neutral component of a scalar doublet of SU(2), $S$. The annihilation mode of $S \bar{S}$ through $s$-channel $Z$ boson exchange is allowed with cross section given by

$$
\langle\sigma(S \bar{S} \rightarrow f \bar{f}) v\rangle=\frac{N_{c} G_{F}^{2}}{2 \pi} \frac{32}{3} \frac{\left(\left|a_{L}\right|^{2}+\left|a_{R}\right|^{2}\right)\left(m_{S} v\right)^{2}}{\left(1-4 m_{S}^{2} / m_{Z}^{2}\right)^{2}}
$$

where $v$ is the velocity of DM, $N_{c}=3(1)$ for quarks (leptons) and $a_{L}\left(a_{R}\right)$ is the coupling of the left-handed (right-handed) fermions to the $Z$ boson. However, the annihilation cross section of dark matter via $Z$ bosons is directly related to the direct detection cross section. In fact, the annihilation of a complex scalar via $s$-channel $Z$ boson exchange has been excluded by direct detection experiments. This connection can be avoided for other types of scalar dark matter. If there is a mass splitting between the scalar and pseudo-scalar component of $S$, the lighter one will be the DM candidate and its scattering off a nucleus via $Z$ boson exchange can be kinematically forbidden, provided that its kinetic energy is less than the mass difference between scalar and pseudo-scalar component of $S$. As the average velocity of dark matter during freeze-out is much larger $\left((v / c)^{2} \sim 1 / 20\right)$ compared to the average local velocity of dark matter $(v \sim 220 \mathrm{~km} / \mathrm{s})$, coannihilation via $s$-channel $Z$ exchange still occurs. Another possibility is to introduce another $S^{\prime}$, a singlet under $\mathrm{SU}(2) \times \mathrm{U}(1)$ with the same $G_{\nu}$ quantum numbers as $S$. We can write a term of form $S^{\prime} H^{\dagger} \cdot S$ which leads to a mixing between $S$ and $S^{\prime}$. The dark matter will be the lighter combination of $S$ and $S^{\prime}$ and its annihilation cross section will then be given by the same formula as $\sigma(S \bar{S} \rightarrow f \bar{f})$ rescaled by a factor of $\sin ^{4} \alpha$ where $\alpha$ is the mixing. Thus, by adjusting $\alpha$, $\sigma(S \bar{S} \rightarrow f \bar{f})$ can be tuned and lead to the correct relic abundance. For $m_{b}<m_{D M}<m_{W}$, a

mixing angle $\sin \alpha=0.5$ and taking a typical velocity at freeze-out of $(v / c)^{2} \sim 1 / 20$, we estimate 
for $m_{S}=60 \mathrm{GeV}$,

$$
\left\langle\sigma_{t o t} v\right\rangle \simeq 3 \times 10^{-26} \frac{\mathrm{cm}^{3}}{\mathrm{~s}} \frac{\left(m_{S} / 60 \mathrm{GeV}\right)^{2}}{\left(1-4\left(m_{S} / 60 \mathrm{GeV}\right)^{2} / m_{Z}^{2}\right)^{2}}\left(\frac{\sin \alpha}{0.5}\right)^{4} .
$$

For higher values of $m_{S},\left\langle\sigma_{t o t} v\right\rangle$ can exceed $3 \times 10^{-26} \mathrm{~cm}^{3} / \mathrm{s}$ especially when new annihilation modes to $t \bar{t}, W^{+} W^{-}$and $Z Z$ open up. For $m_{S} \gg m_{E W}$, we can write

$$
\left\langle\sigma_{t o t} v\right\rangle \simeq 3 \times 10^{-26} \frac{\mathrm{cm}^{3}}{\mathrm{~s}}\left(\frac{1.1 \mathrm{TeV}}{m_{S}}\right)^{2}\left(\frac{\sin \alpha}{0.5}\right)^{4} .
$$

If the dark matter is fermionic and belongs to an $\mathrm{SU}(2)$ doublets, the decay channel through $Z$ boson exchange is open with cross section

$$
\langle\sigma(F \bar{F} \rightarrow f \bar{f}) v\rangle=\frac{4 N_{c} G_{F}^{2}}{\pi} \frac{\left(\left|a_{L}\right|^{2}+\left|a_{R}\right|^{2}\right) M_{F}^{2}}{\left(1-4 m_{F}^{2} / m_{Z}^{2}\right)^{2}} .
$$

Unlike the previous case, there is no $v^{2}$ suppression factor, since the initial state particles are Dirac fermions rather than scalars and the annihilation can be $s$-wave. The cross-section will then exceed

$3 \times 10^{-26} \mathrm{~cm}^{3} / \mathrm{s}$ for $1 \mathrm{GeV}<m_{F}<1 \mathrm{TeV}$. If the dark matter is fermionic and belongs to an $\mathrm{SU}(2)$ doublet, the annihilation cross section of dark matter via $Z$ bosons is directly related to the direct detection cross section. Even an annihilation cross section of $\langle\sigma v\rangle \sim 3 \times 10^{-26} \mathrm{~cm}^{3} / \mathrm{s}$ leads to a direct detection cross section of the order of $10^{-39} \mathrm{~cm}^{2}$ for fermionic DM, which has been excluded by direct detection experiments (see e.g. [23]). Hence, the annihilation via $s$-channel $Z$ boson exchange can only lead to a subdominant contribution of the DM annihilation cross section. This bound can be avoided by introducing a singlet $F^{\prime}$ that mixes with $F$, such that the annihilation cross section is reduced by the mixing analogously to the scalar dark matter case.

\section{Annihilation via $t$-channel $F(S)$ exchange}

Let us consider the case in which $S$ is a singlet that plays the role of dark matter. The annihilation to $l \bar{l}, \nu \bar{\nu}$ via $t$-channel $F$ exchange is helicity suppressed and cannot account for the required total annihilation rate. However, the related three-body decay with the additional emission of a gauge boson, like electromagnetic[24] or electroweak[25] bremsstrahlung can account for the thermal DM annihilation cross section. The annihilation to $\nu \nu$ via the helicity flipping $t$-channel $F$ exchange is suppressed by a lepton number violating coupling and suppressed by the mass of the exchanged fermion $F$, which both also control the smallness of neutrino masses. The cross section ends up to be too small for most regions of parameter space [14], see however [11, 12].

Let us finally discuss if the dark matter is fermionic and belongs to an SU(2) doublets, the dominant annihilation modes can be $F \bar{F} \rightarrow \nu \bar{\nu}, l \bar{l}$ via $t$-channel $S$ exchange. In this case, there is no $p$-wave suppression and the cross section can be of order of

$$
\langle\sigma(F \bar{F} \rightarrow \nu \bar{\nu}, \bar{l}) v\rangle=\frac{g^{4}}{8 \pi} \frac{m_{F}^{2}}{\left(m_{F}^{2}-m_{S}^{2}\right)^{2}},
$$


neglecting the final state masses. Here $g$ denotes a generic Yukawa coupling defined in Eq. (1). Taking $m_{S} \sim m_{F} \sim 100 \mathrm{GeV}, g$ should be of the order of 0.1. With such a large coupling, $\mu \rightarrow e \gamma$ exceeds the experimental bounds unless a specific flavor structure is invoked to suppress this process.

\section{Annihilation via $s$-channel Higgs exchange}

Finally, we give the annihilation via Higgs exchange. Away from the resonant production of the Higgs, the annihilation of $S$ via $s$-channel can be related to the Higgs decay width for a Higgs into a final state $X$

$$
\begin{aligned}
\left\langle\sigma\left(S S \rightarrow h^{*} \rightarrow X\right)_{H} v\right\rangle & =\left.\left(2 m_{h} \Gamma(h \rightarrow X)\right)\right|_{m_{h} \rightarrow 2 M_{S}} \frac{1}{4 M_{S}^{2}} \frac{4|\lambda v|^{2}}{\left(4 M_{S}^{2}-m_{h}^{2}\right)^{2}} \\
& =\frac{\left.\Gamma(h \rightarrow X)\right|_{m_{h} \rightarrow 2 M_{S}}}{M_{S}} \frac{4|\lambda v|^{2}}{\left(4 M_{S}^{2}-m_{h}^{2}\right)^{2}},
\end{aligned}
$$

with the Higgs mass $m_{h}$ and the effective coupling of $S$ to the Higgs $h$ defined by $\mathcal{L} \supset(\lambda v) h S S$. The DM phenomenology is similar to a scalar singlet DM model (see e.g. [26]). In particular, there will be a close correlation between annihilation rate of $S \bar{S}$ and the DM-nucleon cross section and therefore the direct detection rate.

Similarly to Eq. (23), we can calculate the $S$-wave contribution to the annihilation of fermionic dark matter $F$ via $s$-channel Higgs $h$ exchange into a final state $X$

$$
\left\langle\sigma\left(F \bar{F} \rightarrow h^{*} \rightarrow X\right)_{H} v\right\rangle=\left.\left(2 m_{h} \Gamma(h \rightarrow X)\right)\right|_{m_{h} \rightarrow 2 M_{F}} \frac{1}{4 M_{F}^{2}} \frac{2 M_{F}^{2}\left(\left|y_{L}\right|^{2}+\left|y_{R}\right|^{2}\right)}{\left(4 M_{F}^{2}-m_{h}^{2}\right)^{2}},
$$

with the Yukawa coupling $\mathcal{L} \supset-\bar{F}\left(y_{L} P_{L}+y_{R} P_{R}\right) h F$ coupling to the CP even scalar $h$, which leads to a similar phenomenology like the fermionic singlet DM model (see e.g. [27]). As for the scalar case, a relation between the annihilation rate and the direct detection is present.

\section{Conclusions and discussion}

The smallness of neutrino masses is one of the longstanding problems in the phenomenology of particle physics. Various models have been proposed in the literature within which neutrino masses are produced at loop level so their smallness is natural. In this paper, we have discussed the class of models within which neutrino masses are produced at loop level via a Yukawa term that couples neutrinos to new scalars and fermions. We studied and outlined some general results that can be drawn from the topology of neutrino mass diagrams or the symmetry, $G_{\nu}$, imposed on the model. We have discussed conditions on the $G_{\nu}$ symmetry and topology of loop diagrams that forbid the presence of a lower order, and consequently dominating, contribution to the neutrino mass. Under these conditions these diagrams will therefore give the dominant contribution to the neutrino mass. More general results are outlined item by item in sect. 4 . 
In this paper, we have assumed that the $G_{\nu}$ symmetry remains unbroken. In case that the SM particles are invariant under the $G_{\nu}$ symmetry, the lightest new particle with a non-trivial behavior under this symmetry will be stable. If this particle is neutral, it can contribute to the dark matter in the universe. Independently of a given model, there have been studies of the impact of different symmetries on the DM predictions (See e.g. [15-18]). We briefly discuss the implications of the discrete symmetry for dark matter stabilization and discuss the possibility of the existence of multiple dark matter candidates for an abelian group that can be decomposed to the direct product of other groups. We also discussed various possible modes of annihilation of a dark matter pair.

Within the class of models that we have discussed in this paper, the scale of new physics can be as low as the electroweak scale. The new particles that are added have no strong interactions; however, they can have electroweak interactions. The model can include new charged particles coupled to the Higgs which, along with the SM contributions, may explain the possible excess in the diphoton Higgs decay channel [28].

At a hadron collider such as the LHC, the only production mode of these particles is through electroweak interactions but in a lepton collider such as the ILC, these particles can be also produced via Yukawa interactions in Eq. (1) in the $t$-channel. If all the SM particles transform trivially under $G_{\nu}$ and all new particles carry $G_{\nu}$ charges, these particles can be produced at colliders only in association with other new particles such that the final products form a singlet of $G_{\nu}$. For example, if $G_{\nu}=Z_{2}$, the new particles that are odd under $Z_{2}$ can be produced only in pairs. Moreover, the $G_{\nu}$ symmetry implies that the decay products of new particles include lighter new (beyond SM) particles. In fact these particles will go through a chain of successive decays until they produce stable new particle(s). If these final stable products are neutral, they will appear as missing energy but, if they are charged, they can be detected. Since they are heavy and have no strong interactions, they will generally lose energy with a rate smaller than the muon energy loss rate which means they come to rest only after they exit the detector. In this case, the signature of the model will be quite distinct from the SM background, raising the discovery chance of the model.

Decay of the new particles can take place through the Yukawa coupling in Eq. (1) which means each decay produces a lepton along with a new particle. The branching ratio to different flavors is determined by $g_{i j \alpha}$. This is the same coupling that determines the flavor structure of the neutrino mass matrix. In principle, by studying the flavor composition of the decay products of the new particles, one can cross check these models. This possibility has been studied in detail in [29] for the specific case of the SLIM model $[12,13]$ where $G_{\nu}=Z_{2}$ and the new fermions are neutral. Unfortunately, the high rate of background and the uncertainty in luminosity will limit the capability of the LHC to extract the flavor structure of the coupling. However, a lepton collider can have a better chance of determining the coupling. If the model contains multiply charged particles, their production will be enhanced by square of their charge. Moreover their successive decay to multiple charged leptons plus missing energy or new stable charged particle will provide 
a distinct signature enhancing the chances of discovery at lepton and hadron colliders.

One of the key ingredients used in our setup to suppress lower loop contributions is the presence of discrete symmetries at quantum level. Although in our setup discrete symmetries are global and not gauged, there are arguments that all symmetries, including discrete global symmetries, should be gauged in a theory of quantum gravity [30]. This is due to the expectation, mainly advocated in string theory, that all symmetries have a geometric origin and the space-time itself is a locally constructed, secondary concept, in these settings. Thus, all the symmetries should be local symmetries. These symmetries could be broken due to quantum gravity effects and/or anomalies [31]. The effects of symmetry breaking (if there is any) are expected to be suppressed by inverse powers of $m_{P l}$. Based on the dimensional analysis, we can estimate the contribution from the $G_{\nu}$ violating effects to the neutrino mass to be at most $\left(\langle H\rangle^{2} / m_{P l}\right)\left(M_{N E W} / m_{P l}\right)^{n} \sim$ $10^{-6} \mathrm{eV}\left(M_{N E W} / m_{P l}\right)^{n}$ for some $n=0,1,2, \ldots$ Thus, we can safely neglect this effect.

In summary, small neutrino masses can be generated at the loop level in models in which the leptons couple to the new sector. New symmetries guarantee the absence of a Dirac mass term for neutrinos and can forbid lower loop diagrams. The presence of this symmetry leads to neutral stable candidates which might explain the observed dark matter abundance in the Universe. The additional suppression due to the higher loop order allows to lower the scale of new physics down to the TeV scale keeping large couplings and providing specific testable signatures at colliders and observable lepton flavor violating processes.

\section{Acknowledgements}

The authors acknowledge partial support from the European Union FP7 ITN INVISIBLES (Marie Curie Actions, PITN- GA-2011- 289442). They also thank Galileo Galilei Institute for Theoretical Physics for its hospitality. YF is grateful to ICTP for partial financial support and hospitality. She

thanks M. M. Sheikh-Jabbari for useful discussions. MS acknowledges support by the Australian Research Council.

\section{References}

[1] P. Minkowski, Phys. Lett. B67, 421 (1977); T. Yanagida, in Proceedings of the Workshop on The Unified Theory and the Baryon Number in the Universe, edited by O. Sawada and A. Sugamoto (KEK, Tsukuba, Japan, 1979) p. 95; S. L. Glashow, in Proceedings of the 1979 Cargèse Summer Institute on Quarks and Leptons, edited by M. L. vy, J.-L. Basdevant, D. Speiser, J. Weyers, R. Gastmans, and M. Jacob (Plenum Press, New York, 1980) pp. 687-713; M. GellMann, P. Ramond, and R. Slansky, in Supergravity, edited by P. van Nieuwenhuizen and D. Z. Freedman (North Holland, Amsterdam, 1979) p. 315; R. N. Mohapatra and G. Senjanović, Phys. Rev. Lett. 44, 912 (1980). 
[2] A. Zee, Phys. Lett. B93, 389 (1980).

[3] A. Zee, Phys. Lett. B161, 141 (1985); A. Zee, Nucl. Phys. B264, 99 (1986); K. S. Babu, Phys. Lett. B203, 132 (1988).

[4] K. S. Babu and C. N. Leung, Nucl. Phys. B619, 667 (2001), hep-ph/0106054; A. de Gouvea and J. Jenkins, Phys. Rev. D77, 013008 (2008), 0708.1344 [hep-ph]; P. W. Angel, N. L. Rodd, and R. R. Volkas(Dec. 2012), 1212.6111.

[5] K. Babu, S. Nandi, and Z. Tavartkiladze, Phys.Rev. D80, 071702 (2009), arXiv:0905.2710 [hep-ph]; F. Bonnet, D. Hernandez, T. Ota, and W. Winter, JHEP 0910, 076 (2009), arXiv:0907.3143 [hep-ph]; S. Kanemura and T. Ota, Phys.Lett. B694, 233 (Sep. 2010), 1009.3845; Y. Liao, Phys.Lett. B694, 346 (Sep. 2011), 1009.1692.

[6] F. Bonnet, M. Hirsch, T. Ota, and W. Winter, JHEP 1207, 153 (2012), arXiv:1204.5862 [hep-ph].

[7] K. S. Babu and E. Ma, Mod. Phys. Lett. A4, 1975 (1989); E. Ma, Phys. Rev. Lett. 81, 1171 (1998), hep-ph/9805219.

[8] R. Bouchand and A. Merle, JHEP 1207, 084 (May 2012), 1205.0008.

[9] L. M. Krauss, S. Nasri, and M. Trodden, Phys. Rev. D67, 085002 (2003), hep-ph/0210389.

[10] K. Cheung and O. Seto, Phys. Rev. D69, 113009 (2004), hep-ph/0403003; T. Asaka, S. Blanchet, and M. Shaposhnikov, Phys. Lett. B631, 151 (2005), hep-ph/0503065; J. Kubo, E. Ma, and D. Suematsu, Phys. Lett. B642, 18 (2006), hep-ph/0604114; E. J. Chun and H. B. Kim, JHEP 10, 082 (2006), hep-ph/0607076; T. Hambye, K. Kannike, E. Ma, and M. Raidal, Phys. Rev. D75, 095003 (2007), hep-ph/0609228; J. Kubo and D. Suematsu, Phys. Lett. B643, 336 (2006), hep-ph/0610006; K. S. Babu and E. Ma, Int. J. Mod. Phys. A23, 1813 (2008), 0708.3790 [hep-ph]; P.-H. Gu, M. Hirsch, U. Sarkar, and J. W. F. Valle, Phys. Rev. D79, 033010 (2009), 0811.0953 [hep-ph]; N. Sahu and U. Sarkar, Phys. Rev. D78, 115013 (2008), 0804.2072 [hep-ph]; M. Aoki, S. Kanemura, and O. Seto, Phys. Rev. Lett. 102, 051805 (2009), 0807.0361 [hep-ph]; M. Aoki, S. Kanemura, and O. Seto, Phys. Rev. D80, 033007 (2009), 0904.3829 [hep-ph]; T. Hambye, F. S. Ling, L. Lopez Honorez, and J. Rocher, JHEP 07, 090 (2009), 0903.4010 [hep-ph]; E. Ma, Phys.Rev. D80, 013013 (2009), 0904.4450 [hep-ph]; A. Adulpravitchai, M. Lindner, A. Merle, and R. N. Mohapatra, Phys.Lett. B680, 476 (2009), 0908.0470 [hep-ph]; J. March-Russell, C. McCabe, and M. McCullough, JHEP 1003, 108 (2010), arXiv:0911.4489 [hep-ph]; N. Okada and O. Seto, Phys.Rev. D82, 023507 (Feb. 2010), 1002.2525; T. Li and W. Chao, Nucl.Phys. B843, 396 (2011), 1004.0296 [hepph]; D. Meloni, S. Morisi, and E. Peinado, Phys.Lett. B697, 339 (Nov. 2011), 1011.1371; M. Hirsch, S. Morisi, E. Peinado, and J. Valle, Phys.Rev. D82, 116003 (Jul. 2010), 1007.0871 [hep-ph]; A. Adulpravitchai, P.-H. Gu, and M. Lindner, Phys.Rev. D82, 073013 (Jul. 2010), 1007.1115 [hep-ph]; S. Kanemura, O. Seto, and T. Shimomura, Phys.Rev. D84, 016004 (Jan. 
2011), 1101.5713 [hep-ph]; W.-F. Chang and C.-F. Wong, Phys.Rev. D85, 013018 (2012), 1104.3934 [hep-ph]; M. Lindner, D. Schmidt, and T. Schwetz, Phys.Lett. B705, 324 (May 2011), 1105.4626 [hep-ph]; S. Kanemura, T. Nabeshima, and H. Sugiyama, Phys.Rev. D85, 033004 (2012), arXiv:1111.0599 [hep-ph]; Y. Ahn and H. Okada, Phys.Rev. D85, 073010 (Jan. 2012), 1201.4436; W. Chao(Feb. 2012), 1202.6394.

[11] E. Ma, Phys. Rev. D73, 077301 (2006), hep-ph/0601225.

[12] C. Boehm, Y. Farzan, T. Hambye, S. Palomares-Ruiz, and S. Pascoli, Phys. Rev. D77, 043516 (2008), hep-ph/0612228.

[13] Y. Farzan, Phys. Rev. D80, 073009 (2009), 0908.3729 [hep-ph].

[14] Y. Farzan, S. Pascoli, and M. A. Schmidt, JHEP 1010, 111 (May 2010), 1005.5323.

[15] E. Ma, Phys. Lett. B662, 49 (2008), 0708.3371 [hep-ph].

[16] B. Batell, Phys.Rev. D83, 035006 (Jul. 2011), 1007.0045.

[17] A. Adulpravitchai, B. Batell, and J. Pradler, Phys.Lett. B700, 207 (Mar. 2011), 1103.3053.

[18] G. Belanger, K. Kannike, A. Pukhov, and M. Raidal, JCAP 1204, 010 (Feb. 2012), 1202.2962.

[19] K. S. Babu and E. Ma, Phys. Rev. Lett. 61, 674 (1988).

[20] E. Ma, Phys.Rev. D82, 037301 (2010), 1006.3524 [hep-ph].

[21] L. Lavoura, Eur. Phys. J. C29, 191 (2003), hep-ph/0302221.

[22] W. Buchmüller and T. Yanagida, Phys. Lett. B445, 399 (1999), hep-ph/9810308; F. Vissani, JHEP 11, 025 (1998), hep-ph/9810435; R. Barbieri, L. J. Hall, D. R. Smith, A. Strumia, and N. Weiner, JHEP 12, 017 (1998), hep-ph/9807235.

[23] E. Aprile et al. (XENON100 Collaboration), Phys.Rev.Lett. 109, 181301 (2012), arXiv:1207.5988 [astro-ph.CO].

[24] L. Bergstrom, Phys.Lett. B225, 372 (1989).

[25] N. F. Bell, J. B. Dent, T. D. Jacques, and T. J. Weiler, Phys.Rev. D83, 013001 (2011), arXiv:1009.2584 [hep-ph]; N. F. Bell, J. B. Dent, A. J. Galea, T. D. Jacques, L. M. Krauss, et al., Phys.Lett. B706, 6 (2011), arXiv:1104.3823 [hep-ph].

[26] V. Silveira and A. Zee, Phys.Lett. B161, 136 (1985).

[27] Y. G. Kim, K. Y. Lee, and S. Shin, JHEP 0805, 100 (2008), arXiv:0803.2932 [hep-ph].

[28] M. Carena, I. Low, and C. E. Wagner, JHEP 1208, 060 (Jun. 2012), 1206.1082.

[29] Y. Farzan and M. Hashemi, JHEP 1011, 029 (Sep. 2010), 1009.0829. 
[30] L. E. Ibanez and G. G. Ross, Phys. Lett. B260, 291 (1991); T. Banks and M. Dine, Phys. Rev. D45, 1424 (1992), hep-th/9109045.

[31] T. Araki, Prog. Theor. Phys. 117, 1119 (2007), hep-ph/0612306; T. Araki et al., Nucl. Phys. B805, 124 (2008), 0805.0207 [hep-th]. 